\title{
Poly(ethylene glycol)-induced acceleration of free radical polymerization of methyl methacrylate: effects of highly viscous solvent and kinetic study
}

\author{
Shin-ichi Matsuoka, Takashi Kikuno, Koji Takagi and Masato Suzuki
}

Poly(ethylene glycol) with a molecular weight of 400 (PEG400) as an environmentally benign and highly viscous polymeric solvent was found to effectively accelerate the free radical polymerization (FRP) of methyl methacrylate and to afford a highmolecular-weight polymer. When the polymerization in PEG400 was performed at a monomer concentration of $3.0 \mathrm{~mol} \mathrm{I}^{-1}$ and an initiator $\left(2,2^{\prime}\right.$-azobis(isobutyronitrile)) concentration of $0.3 \times 10^{-2} \mathrm{moll}^{-1}$, the monomer conversion was completed for $6 \mathrm{~h}$ to afford a polymer with a number-average molecular weight $\left(M_{n}\right)$ of 838000 . After polymerization, the PEG400 solvent was readily recovered and reused. The solvent effects of highly viscous PEG400 were kinetically analyzed. The polymerization rate $\left(\boldsymbol{R}_{\mathrm{p}}\right)$ was proportional to the monomer concentration and the square root of both the initiator concentration and the viscosity of the polymerization media $(\eta)$. For the FRP in the mixed solvents of PEG400 and toluene, both $R_{\mathrm{p}}$ and $M_{\mathrm{n}}$ values increased linearly with the square root of the $\eta$ value. The kinetic study has shown that the highly viscous PEG400 solvent largely suppresses diffusion-controlled bimolecular termination.

Polymer Journal (2010) 42, 368-374; doi:10.1038/pj.2010.17; published online 17 March 2010

Keywords: diffusion-controlled termination; environmentally benign solvent; free radical polymerization; kinetic study; methyl methacrylate; poly(ethylene glycol); viscosity effect

\section{INTRODUCTION}

Chemical transformations in an environmentally benign alternative solvent are of growing interest, because volatile organic solvents for synthetic chemistry usually have toxicity and flammability. Although use of nonvolatile organic solvents may cause a separation problem, it can reduce environmental risks. Liquid polymers such as silicone oil, liquid paraffin, poly(ethylene glycol) (PEG) and several oligomers are examples of the most accessible nonvolatile organic solvents, and they can be used to form a highly viscous solution. Among them, PEG with low molecular weight is a versatile nonvolatile solvent for organic reactions because of good solubility of organic substrates in PEG. The PEG solvent offers advantages over conventional organic solvents in terms of low toxicity, low flammability and biodegradability. Recently, PEGs have been used for a variety of organic reactions such as the Suzuki coupling, ${ }^{1-6}$ the Michael addition, ${ }^{7}$ hydrogenation, ${ }^{8-11}$ and so on. ${ }^{12-19}$ Perrier et al.$^{20}$ reported the first use of PEG as a polymerization solvent. They presume that the polarity and metal coordination ability of PEG would promote copper-catalyzed living radical polymerization of methyl methacrylate (MMA) and styrene.

In free radical polymerization (FRP), propagating radicals usually terminate through bimolecular reactions. Such a termination reaction is under diffusion control, so that the termination rate constant $\left(k_{\mathrm{t}}\right)$ is inversely proportional to the viscosity of the polymerization system $(\eta)$. As such, by substituting $k_{\mathrm{t}, 0} / \eta\left(k_{\mathrm{t}, 0}=\right.$ termination rate constant when $\eta=1 \mathrm{cP}$ ) for $k_{\mathrm{t}}$ in conventional FRP kinetics, the polymerization rate $\left(R_{\mathrm{p}}\right)$ can be expressed as Equation (1): ${ }^{21,22}$

$$
R_{\mathrm{p}}=k_{\mathrm{p}} \sqrt{\frac{k_{\mathrm{d}} f}{k_{t, 0}}}[\mathrm{M}] \sqrt{[\mathrm{I}]} \sqrt{\eta}
$$

where $k_{\mathrm{p}}$ is the propagation rate constant, $k_{\mathrm{d}}$ and $f$ are the rate constant and the efficiency for decomposition of the initiator, respectively, and $[\mathrm{M}]$ and $[\mathrm{I}]$ are the concentrations of monomer and initiator, respectively. The number-average degree of polymerization $\left(\bar{P}_{\mathrm{n}}\right)$ is generally given by Equation (2) when chain transfer reactions are negligible: ${ }^{23,24}$

$$
\bar{P}_{\mathrm{n}}=\frac{k_{\mathrm{p}}^{2}[\mathrm{M}]^{2}}{k_{\mathrm{t}} R_{\mathrm{P}}}
$$

Substituting Equation (1) and $k_{\mathrm{t}}=k_{\mathrm{t}, 0} / \eta$ into Equation (2) gives Equation (3).

$$
\bar{P}_{\mathrm{n}}=\frac{k_{\mathrm{p}}}{\sqrt{k_{\mathrm{t}, 0} k_{\mathrm{d} f}}} \frac{[\mathrm{M}] \sqrt{\eta}}{\sqrt{[\mathrm{I}]}}
$$


Equations ( 1 and 3 ) show that both $R_{\mathrm{p}}$ and $\bar{P}_{\mathrm{n}}$ are proportional to the square root of $\eta$. Thus, highly viscous media is expected to accelerate FRP from the initial stage and to afford a high-molecular-weight polymer. Since the first report of the gel effect, ${ }^{25}$ the diffusion-controlled termination in radical polymerization has been intensely investigated. ${ }^{26-29}$ It was reported that the FRP of MMA rapidly proceeds in a highly viscous solution of polystyrene.$^{30}$ Recently, several research groups have investigated the FRPs of methacrylates in ionic liquids as highly viscous nonvolatile solvents and found that polymerizations are accelerated to produce high-molecular-weight polymers. ${ }^{31-35}$ They have proposed several solvent effects such as high viscosity and polarity of ionic liquids, ${ }^{31-34}$ and domain separation. ${ }^{35}$ Although ionic liquids can be versatile solvents for FRP, the very high cost and the unknown toxicity are their weak points. Thus, we are prompted to develop a new FRP system by using conventional and commercially available nonvolatile solvents.

We herein report the first use of PEG as a highly viscous, readily available and environmentally benign solvent for FRP of MMA. It was found that the PEG solvent accelerates the FRP to yield a highmolecular-weight poly(methyl methacrylate) (PMMA). To examine the effects of the highly viscous solvent, the FRP system has been kinetically studied; both $R_{\mathrm{p}}$ and $M_{\mathrm{n}}$ are proportional to the square root of $\eta$, as presented in Equations (1 and 3).

\section{EXPERIMENTAL PROCEDURE}

\section{Materials}

MMA (Wako Pure Chemical Industries, Tokyo, Japan, >97\%) was washed with an aqueous $5 \% \mathrm{NaOH}$ solution and then with water, dried over $\mathrm{MgSO}_{4}$ and distilled from $\mathrm{CaH}_{2}$ under reduced pressure. 2,2'-Azobis(isobutyronitrile) (AIBN) (Kanto Chemical, Tokyo, Japan, 97\%) was purified by recrystallization from methanol. PEGs with average molecular weights of 200 (polyethylene glycol 200 , Kanto, $>90 \%$, molecular weight of 190-210), 400 (polyethylene glycol 400, Kanto, $>90 \%$, molecular weight of 380-420), 1000 (polyethylene glycol 1000, Kanto, $>90 \%$, molecular weight of 950-1050) and 8000 (polyethylene glycol 6000 , Kanto, $>90 \%$, molecular weight of 7300-10 200) were used as received. Toluene (Kanto, $>99 \%$ ) was distilled from sodium before use.

\section{Polymerization}

In a three-necked flask equipped with a three-way stopcock, PEG400 (30 g, $26.5 \mathrm{ml}$ ) was stirred under vacuum to remove dissolved oxygen, and then MMA (3g, $29.9 \mathrm{mmol}$ ) and AIBN (49 mg, $0.298 \mathrm{mmol}$ ) were added under nitrogen atmosphere. When using the mixed solvent of PEG400 and toluene, the polymerization system was degassed by three freeze-vacuum-thaw cycles. The mixture was mechanically or magnetically stirred at $60^{\circ} \mathrm{C}$ for $6 \mathrm{~h}$ under nitrogen atmosphere. Water or methanol $(150 \mathrm{ml})$ was poured into the polymerization solution and the subsequent filtration afforded PMMA $(2.85 \mathrm{~g})$ in $95 \%$ yield. In cases in which the polymerization rate was estimated, aliquots of the polymerization solution were sampled several times during polymerization and subjected to ${ }^{1} \mathrm{H}$ nuclear magnetic resonance spectroscopy, which informed the monomer conversion by the signal integral ratio between $\mathrm{CH}_{2}=\mathrm{C}$ of MMA and $\mathrm{C}-\mathrm{CH}_{3}$ of PMMA.

\section{Measurements}

Number-average molecular weight $\left(M_{\mathrm{n}}\right)$ and molecular weight distribution $\left(M_{\mathrm{w}} / M_{\mathrm{n}}\right)$ were determined by size exclusion chromatography in tetrahydrofuran at $40^{\circ} \mathrm{C}$ on four tandem polystyrene gel columns (TSKgel G3000H $\mathrm{HL}_{\mathrm{XL}}$, G4000 $\mathrm{H}_{\mathrm{XL}}$, $\mathrm{G} 5000 \mathrm{H}_{\mathrm{XL}}$ and $\mathrm{G} 6000 \mathrm{H}_{\mathrm{XL}}$, TOSOH, Tokyo, Japan) calibrated against polystyrene standards at a flow rate of $1 \mathrm{ml} \mathrm{min}{ }^{-1}$ with refractive index detection. ${ }^{1} \mathrm{H}$ and ${ }^{13} \mathrm{C}$ nuclear magnetic resonance spectra were recorded on a Bruker Avance-200 $\left(200 \mathrm{MHz}\right.$ for $\left.{ }^{1} \mathrm{H}\right)$ or a Bruker Avance-600 $\left(600 \mathrm{MHz}\right.$ for $\left.{ }^{1} \mathrm{H}\right)$ spectrometer (Bruker BioSpin, Kanagawa, Japan) in $\mathrm{CDCl}_{3}$. Tacticities of PMMAs were determined from $\alpha$-methyl hydrogen signals. Viscosity was measured by sine-wave vibro viscometer SV-10 with two gold-coated transducers and a temperature sensor (A\&D Ltd, Tokyo, Japan).

\section{RESULTS AND DISCUSSION}

We first carried out the FRP of MMA in PEG to high conversion under a variety of conditions as shown in Table 1 to demonstrate the synthetic utility. We then performed kinetic studies of FRP in the initial stage to understand the solvent effects and mechanistic aspects; the effects of polymerization conditions on the $R_{\mathrm{p}}$ and $M_{\mathrm{n}}$ of the resulting PMMA were investigated.

\section{Polymerization results}

To examine the effect of the molecular weights of PEG, the FRPs of MMA with AIBN at $60^{\circ} \mathrm{C}$ were conducted in PEGs with molecular

Table 1 Free radical polymerization of MMA with AIBN

\begin{tabular}{|c|c|c|c|c|c|c|c|c|c|c|}
\hline Run & Solvent & $\begin{array}{l}{[\mathrm{MMA}]} \\
\left(\mathrm{mol}^{-1}\right)\end{array}$ & $\begin{array}{c}10^{2}[\mathrm{AIBN}] \\
\left(\mathrm{mol} / \mathrm{I}^{-1}\right)\end{array}$ & $\eta^{\mathrm{a}}(c P)$ & $\begin{array}{c}\text { Temperature } \\
\left({ }^{\circ} \mathrm{C}\right)\end{array}$ & $\begin{array}{c}10^{5} R_{\mathrm{p}}^{\mathrm{b}} \\
\left(\mathrm{mol}^{-1} \mathrm{~s}^{-1}\right)\end{array}$ & $\begin{array}{l}\text { Time } \\
\text { (h) }\end{array}$ & $\begin{array}{c}\text { Yield } \\
(\%)\end{array}$ & $10^{-3} M_{n}{ }^{c}$ & $M_{\mathrm{w}} / M_{\mathrm{n}}{ }^{\mathrm{d}}$ \\
\hline 1 & PEG200 & 1.0 & 1.0 & 9.62 & 60 & - d $^{d}$ & 6 & 100 & 101 & 4.68 \\
\hline 2 & PEG400 & 1.0 & 1.0 & 14.5 & 60 & 11.1 & 6 & 95 & 177 & 3.12 \\
\hline 3 & PEG1000 & 1.0 & 1.0 & 37.9 & 60 & 9.62 & 6 & 92 & 132 & 2.33 \\
\hline 4 & PEG8000 & 1.0 & 1.0 & 1680 & 60 & 20.5 & 6 & 99 & 113 & 2.13 \\
\hline 5 & PEG400 & 1.0 & 0.20 & 14.5 & 60 & 5.10 & 6 & 87 & 425 & 1.94 \\
\hline 6 & PEG400 & 1.0 & 1.0 & 14.5 & 60 & 11.1 & 1 & 39 & 278 & 2.74 \\
\hline 7 & PEG400 & 3.0 & 3.0 & 5.27 & 60 & 25.2 & 0.5 & 24 & 186 & 2.33 \\
\hline 8 & PEG400 & 3.0 & 3.0 & 5.27 & 60 & 25.2 & 3 & 99 & 222 & 3.00 \\
\hline 9 & PEG400 & 3.0 & 0.30 & 5.27 & 60 & 10.7 & 6 & 99 & 838 & 1.79 \\
\hline 10 & PEG400 & 1.0 & 1.0 & 13.1 & 70 & 25.6 & 6 & 100 & 74 & 4.14 \\
\hline 11 & PEG400 & 1.0 & 1.0 & 11.1 & 80 & 47.2 & 4 & 98 & 21.1 & 2.74 \\
\hline 12 & Toluene & 1.0 & 1.0 & 0.60 & 60 & 2.33 & 24 & 82 & 8.8 & 2.25 \\
\hline 13 & Toluene & 3.0 & 0.30 & 0.60 & 60 & 3.88 & 26 & 35 & 48.6 & 2.63 \\
\hline 14 & Bulk & 9.4 & 0.94 & 0.63 & 60 & 20.8 & 6 & 92 & 143 & 4.19 \\
\hline
\end{tabular}

Abbreviations: AIBN, 2,2'-azobis(isobutyronitrile); MMA, methyl methacrylate; PEG200, poly(ethylene glycol) with molecular weight of 200; PEG400, poly(ethylene glycol) with molecular weight of 400; PEG8000, poly(ethylene glycol) with molecular weight of 8000 .

alnitial viscosity of the polymerization media at the polymerization temperature.

bolymerization rate (conversion $<10 \%$ ).

Polymerization rate (conversion $<10 \%$ ).
'By size exclusion chromatography in tetrahydrofuran using polystyrene standards.

dThe polymerization mixture was heterogeneous. 
weights of 200 (PEG200), 400 (PEG400), 1000 (PEG1000) and 8000 (PEG8000) (Table 1). For the FRPs in PEG400, PEG1000 and PEG8000, the solutions became cloudy after $30 \mathrm{~min}$ and extremely viscous along with the progress of the polymerization without formation of precipitated PMMA. In contrast, the FRP in PEG200 proceeded heterogeneously, precipitating PMMA even at the initial stage. PMMAs were collected by the addition of a large excess of water into the polymerization solution and subsequent filtration. However, the resultant PMMAs were slightly contaminated with the PEG solvent, which was completely excluded by reprecipitation into a large amount of water from tetrahydrofuran. Although the product isolation from a nonvolatile solvent is generally more difficult compared with volatile counterparts, the PMMAs were readily separated from nonvolatile PEG solvent because of large differences in solubility in water between PEG and PMMA. Absence of contamination of the obtained PMMA with PEG also supports the fact that a block copolymer is not formed from them by chain transfer reactions to PEG.

The FRPs in PEG200, PEG400, PEG1000 and PEG8000 for $6 \mathrm{~h}$ almost quantitatively produce PMMAs with much higher molecular weights than those in toluene for $24 \mathrm{~h}$ (runs 1-4, 12). This particular solvent effect of PEG prompted us to investigate further details, for which the experiments were performed in PEG400 because of the following reasons: the size exclusion chromatography profiles of the PMMAs obtained in PEG400, PEG1000 and PEG8000 are unimodal, whereas the heterogeneous polymerization in PEG200 produces PMMA with a multimodal profile and relatively larger polydispersity (Supplementary Figure S1). Thus, the heterogeneous FRP in PEG200 is not suitable for the mechanistic study. Use of PEG1000 and PEG8000, which are solids at room temperature, is not preferable in terms of handling as the solvent and separation of PMMA from the solvent. The $\eta$ value of the PEG8000 polymerization system is almost 100 times as large as that of the PEG400 system. If this high viscosity reduces only the bimolecular termination rate, Equation (1) allows us to calculate the estimated $R_{\mathrm{p}}$ value in PEG8000 from the experimental $R_{\mathrm{p}}$ value in PEG400. In fact, the experimental value of $20.5 \times 10^{-5} \mathrm{moll}^{-1} \mathrm{~s}^{-1}$ (run 4) is much lower than the estimated value of $119 \times 10^{-5} \mathrm{moll}^{-1} \mathrm{~s}^{-1}$. In addition, the $R_{\mathrm{p}}$ value in PEG1000 is slightly lower than that in PEG400, although the viscosity of the PEG1000 solution is twice higher than that of PEG400. Furthermore, FRP gives a relatively higher molecular weight PMMA in PEG400 than in PEG1000 and PEG8000 (runs 2-4). It is known that in the later stage of bulk polymerization, an extremely viscous system, the propagation rate and the initiator efficiency decrease. ${ }^{24,36,37}$ As the highly viscous polymerization solutions of PEG8000 and PEG1000 are comparable to the later stage of the bulk system, such negative factors would affect FRP results and make them inconsistent with the theoretical kinetics (Equations (1 and 3)). Therefore, we suggest that the viscosities of PEG8000 and PEG1000 are too high to effectively promote FRPs.

The FRP at $60^{\circ} \mathrm{C}$ produced the higher molecular weight PMMA as compared with that at 70 and $80^{\circ} \mathrm{C}$ because of the low radical concentration at the relatively low temperature (runs 2, 10 and 11). Thus, the following detailed study was performed at $60{ }^{\circ} \mathrm{C}$.

The time-conversion plots shown in Figure 1 indicate that the FRP proceeded much faster in PEG400 than in toluene. At the monomer concentration of $3.0 \mathrm{moll}^{-1}$, the FRP was accelerated in the region over $\sim 30 \%$ conversion, and completed in $3 \mathrm{~h}$ (run 8 , Figure 1a). In contrast, the FRP mixtures in bulk ${ }^{36-40}$ and in ionic liquids ${ }^{34,35}$ are known to solidify without the complete monomer conversion (see also run 14$)$. The value of $\ln \left([\mathrm{MMA}]_{0} /[\mathrm{MMA}]\right)$ versus polymerization

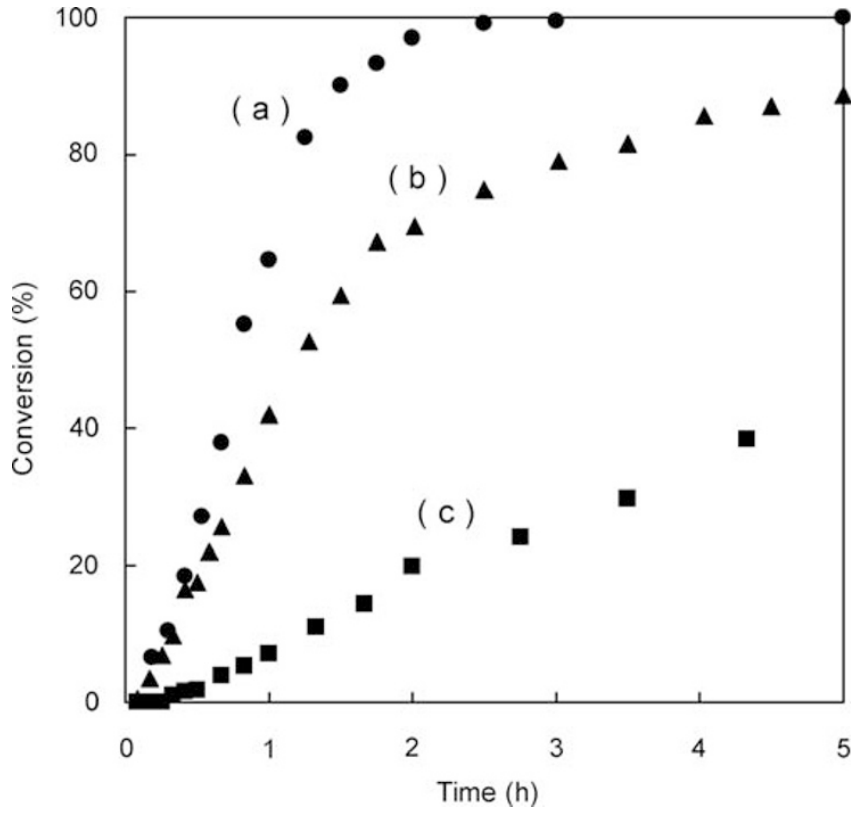

Figure 1 Time versus conversion plots for the polymerization of MMA with AIBN at $60^{\circ} \mathrm{C}$ : (a) run 7, 8; (b) run 2, 6; (c) run 12 in Table 1.

time was linearly fitted on the kinetic plots, showing that $R_{\mathrm{p}}$ is proportional to the first order of the monomer concentration (Supplementary Figure $\mathrm{S} 2$ ). Thus, the $R_{\mathrm{p}}$ values under various polymerization conditions were evaluated from these kinetic plots.

The $R_{\mathrm{p}}$ values in PEG400 in the initial stage of polymerization at initial monomer concentrations of 1.0 and $3.0 \mathrm{moll}^{-1}$ were 4.8 and 2.8 times as large as those in toluene, respectively (runs 2, 9, 12 and 13). Interestingly, these values are almost identical with the square roots of the multiple numbers of $\eta$ values; the viscosities in PEG400 in these monomer concentrations were 24 and 8.8 times as high as those in toluene, respectively. This is in good agreement with Equation (1).

The molecular weights of the PMMAs obtained in PEG400 were roughly 20 times higher than those obtained in toluene for both monomer concentrations (runs 2, 9, 12 and 13). The $M_{\mathrm{n}}$ value was affected by the initial monomer concentration and monomer conversion (runs 2, 6, 7 and 8). Specifically, it decreased with the progress of the polymerization at an initial monomer concentration of $1.0 \mathrm{moll}^{-1}$, but, in contrast, increased gradually in the case of an initial monomer concentration of $3.0 \mathrm{moll}^{-1}$ (Supplementary Figure S3). This difference is presumably due to the gel effect given by the increased viscosity at the relatively high monomer concentration. Even at a low initiator concentration of $0.3 \times 10^{-2} \mathrm{moll}^{-1}$ with a monomer concentration of $3.0 \mathrm{moll}^{-1}$, the FRP proceeded rapidly and completed for $6 \mathrm{~h}$, producing the very high-molecular-weight PMMA with $M_{\mathrm{n}}$ of 838000 and $M_{\mathrm{w}} / M_{\mathrm{n}}$ of 1.79 (run 9). Accordingly, the present synthetic procedure offers a practical advantage for the facile preparation of high-molecular-weight PMMA without chain transfer reactions to PEG400.

These promising results encouraged us to perform further studies, including studies on kinetics. The viscosity of the reaction mixture varies with the progress of polymerization, and the temperature of the polymerization solution may increase at the onset of the gel effect. Thus, the following experiments were conducted at the initial stage of the FRP. 


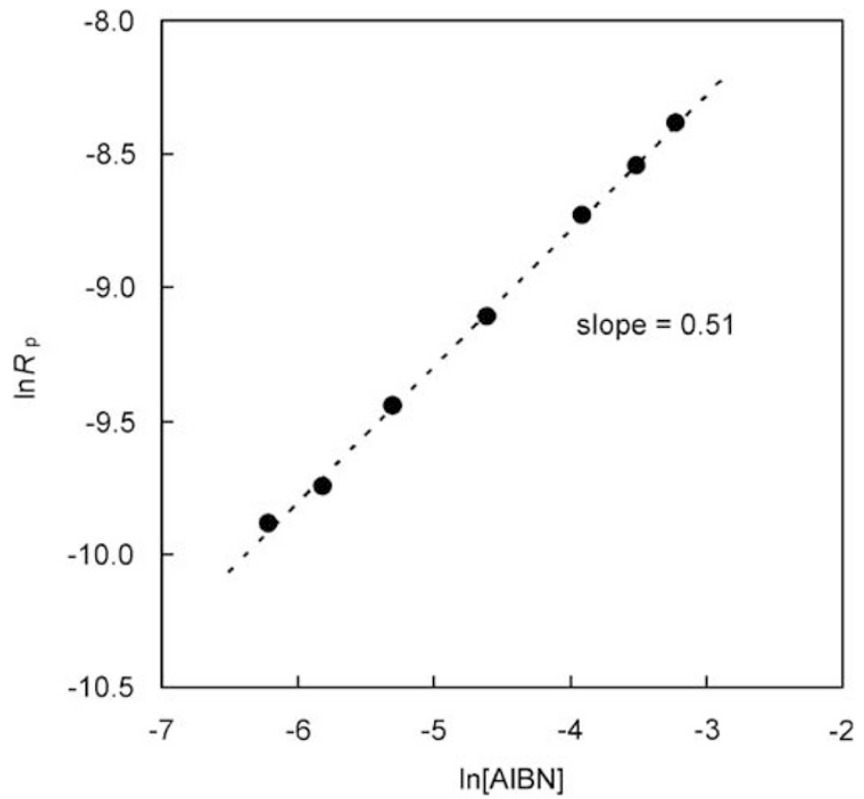

Figure 2 Plots of $\ln R_{\mathrm{p}}$ versus $\operatorname{In}[\mathrm{AIBN}]$ for the polymerization of MMA with AIBN at $60^{\circ} \mathrm{C}$ : $[M M A]=1.0 \mathrm{moll}^{-1}$

\section{Effects of initiator concentration}

Values of $R_{\mathrm{p}}$ were measured at various initiator concentrations ranging from $0.2 \times 10^{-2}$ to $4 \times 10^{-2} \mathrm{moll}^{-1}$ to examine the termination mechanism. A linear relationship between $\ln [\mathrm{AIBN}]$ and $\ln R_{\mathrm{p}}$ was observed with a slope value of 0.51 , indicating that the $R_{\mathrm{p}}$ is proportional to the square root of the initiator concentration (Figure 2). This relationship, in common with the usual solution FRP of MMA, suggests that bimolecular terminations are dominantly involved. By contrast, it is known that single-molecular terminations take place in extremely viscous media, such as a later stage of bulk polymerization, ${ }^{41}$ and in a methanol/water system for dispersion polymerization. ${ }^{42}$ These termination processes are attributed to the lack of mobility of polymer radicals and precipitation of the produced polymer, respectively. The $R_{\mathrm{p}}$ values in these cases are proportional to the $0.6-0.8$ th order of the initiator concentration. On the other hand, the diffusion of polymer chains in PEG400 is not completely suppressed, and polymer radicals have enough mobility to allow bimolecular collisions.

The molecular weights of PMMAs obtained at monomer conversions of $<10 \%$ were examined by varying the initiator concentration. As shown in Figure 3a, when the initiator concentration is decreased, the $M_{\mathrm{n}}$ value increases and reaches 258000 at the very low initiator concentration of $0.3 \times 10^{-2} \mathrm{moll}^{-1}$ with almost constant $M_{\mathrm{w}} / M_{\mathrm{n}}$ values. Expectedly, there is a linear relationship between the $M_{\mathrm{n}}$ value and the reciprocal square root of the initiator concentration as shown in Figure 3c, which is in good agreement with Equation (3). Thus, the molecular weight of the resulting PMMA can be controlled by the initiator concentration at least in the range between $0.3 \times 10^{-2}$ and $18 \times 10^{-2} \mathrm{moll}^{-1}$.

\section{Effects of monomer concentration}

The FRPs were carried out at various monomer concentrations ranging from 0.3 to $9.4 \mathrm{moll}^{-1}$. The resultant relationships of $R_{\mathrm{p}}$ and $\eta$ to the monomer concentration are plotted in Figure 4. The $R_{\mathrm{p}}$ value increases linearly with increasing the monomer concentration

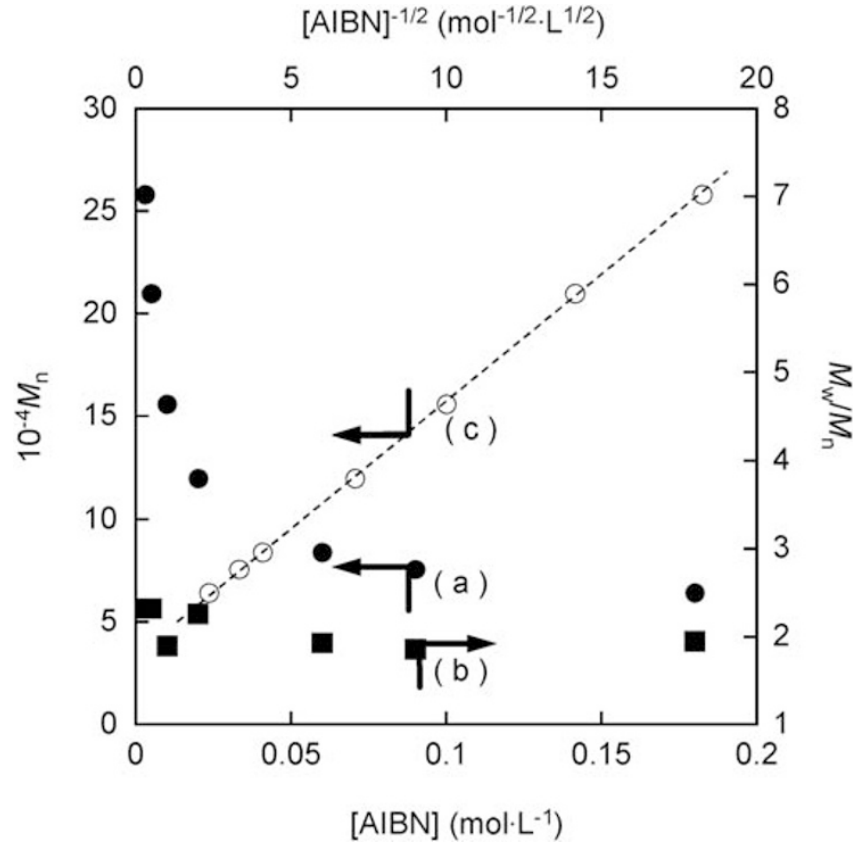

Figure 3 The $M_{\mathrm{n}}$ and $M_{\mathrm{w}} / M_{\mathrm{n}}$ values of PMMA obtained by polymerization in PEG400 with AIBN at $60^{\circ} \mathrm{C}$ : $[\mathrm{MMA}]=1.0 \mathrm{moll}^{-1}$; (a) $10^{-4} \mathrm{M}_{\mathrm{n}}$ versus [AIBN]; (b) $M_{\mathrm{w}} / M_{\mathrm{n}}$ versus $[\mathrm{AIBN}]$; (c) $10^{-4} M_{\mathrm{n}}$ versus $[\mathrm{AIBN}]^{-1 / 2}$.

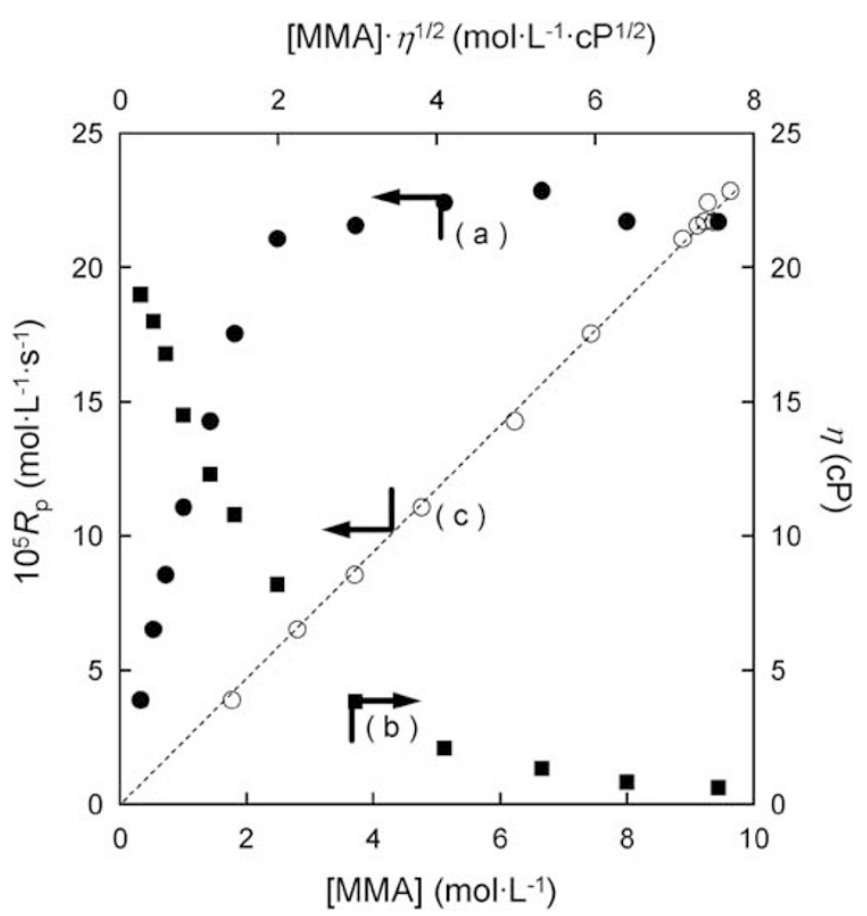

Figure $4 R_{\mathrm{p}}$ and $\eta$ values in the polymerization of MMA in PEG400 with AIBN at $60^{\circ} \mathrm{C}$ : $[\mathrm{AIBN}]=1.0 \times 10^{-2} \mathrm{~mol} \mathrm{I}^{-1}$; (a) $R_{\mathrm{p}}$ versus [MMA], (b) $\eta$ versus [MMA], (c) $R_{\mathrm{p}}$ versus [MMA] $\eta^{1 / 2}$.

in the range of $<2.5 \mathrm{moll}^{-1}$ and then becomes almost constant (Figure 4a). It is obvious that the $\eta$ value decreases when the monomer concentration is increased because of the much lower viscosity of MMA than PEG400 (Figure 4b). Thus, the variable $\eta$ 


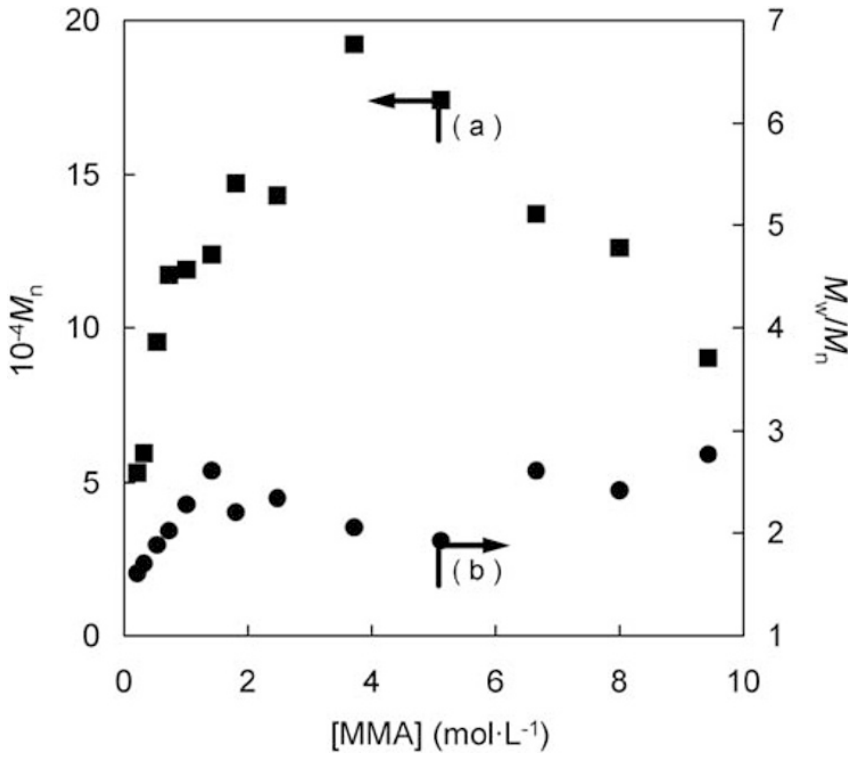

Figure 5 The $M_{\mathrm{n}}$ and $M_{\mathrm{w}} / M_{\mathrm{n}}$ values of PMMA obtained by the polymerization in PEG400 with AIBN at $60^{\circ} \mathrm{C}$ : $[A I B N]=1.0 \times 10^{-2} \mathrm{moll}^{-1}$; (a) $10^{-4} \mathrm{M}_{\mathrm{n}}$ versus [MMA]; (b) $M_{\mathrm{w}} / M_{\mathrm{n}}$ versus [MMA].

values should be considered for this kinetic study. When we plot $R_{\mathrm{p}}$ against $[\mathrm{MMA}] \eta^{1 / 2}$ as shown in Figure $4 c$, the data points fall on a straight line that passes through the origin. This relationship clearly proves Equation (1). Furthermore, this is also supported by the linear plots of $R_{\mathrm{p}} /\left[\mathrm{MMA}\right.$ against $\eta^{1 / 2}$ and of $R_{\mathrm{p}} / \eta^{1 / 2}$ against [MMA] (Supplementary Figure $\mathrm{S} 4$ ).

Figure 5 shows the dependency of the polymer molecular weight on the monomer concentration at the initial stage of the FRP with an initiator concentration of $1.0 \times 10^{-2} \mathrm{moll}^{-1}$. The $M_{\mathrm{n}}$ value increases with increasing monomer concentration in the range of $<4 \mathrm{moll}^{-1}$ and then decreases gradually. The PEG400 solvent system is more efficient to produce high-molecular-weight PMMA than the bulk polymerization $\left([\mathrm{MMA}]=9.44 \mathrm{moll}^{-1}\right)$, and the monomer concentrations of 2-5 $\mathrm{moll}^{-1}$ are appropriate for such a purpose. Equation (3) suggested to us to plot $M_{\mathrm{n}}$ against [MMA] $\eta^{1 / 2}$, but a linear relationship was not clearly observed. The chain transfer reaction rate to MMA and PEG400 would be different. Thus, the deviation from a linear relationship is probably because the monomer concentration affected the frequency of chain transfer reactions.

\section{Effects of viscosity}

To gain additional insights into this kinetic study, FRPs were carried out in a variety of PEG400-toluene mixed solvents that can control the $\eta$ values ranging from 0.60 to $14.5 \mathrm{cP}$ without changing the monomer and initiator concentrations. All FRPs in the mixed solvents homogeneously proceeded with the first order with respect to the monomer concentration (Supplementary Figure S5). Increasing the proportion of PEG400 in the mixed solvent causes the $\eta$ and $R_{\mathrm{p}}$ values to increase (Supplementary Figure S6). The plot of $\ln R_{\mathrm{p}}$ versus $\ln \eta$ was found to show a linear relationship with a slope value of 0.49 (Figure 6), and therefore the plot of $R_{\mathrm{p}}$ versus $\eta^{1 / 2}$ is a good fit for the straight line that passed through the origin (Supplementary Figure S7). These data also clearly support Equation (1).

The $M_{\mathrm{n}}$ of the PMMAs obtained at monomer conversions $<10 \%$ also increased from 16000 to 119000 with increase in the $\eta$ value, and the $M_{\mathrm{w}} / M_{\mathrm{n}}$ values were within 1.7-2.3 (Figure 7). The slope value of

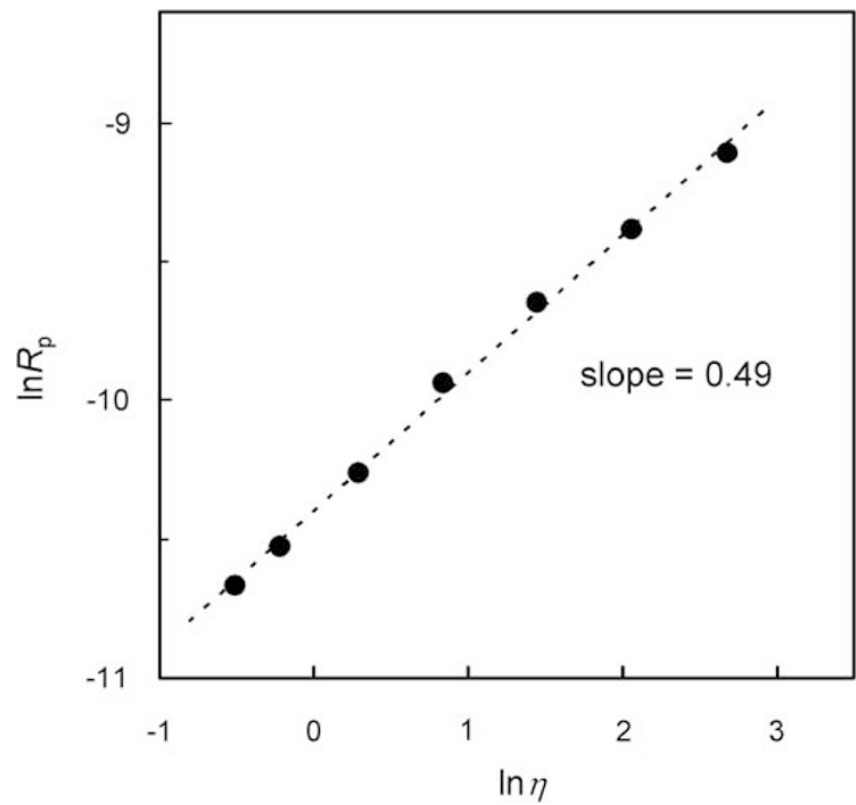

Figure 6 Plots of $\ln R_{\mathrm{p}}$ versus $\ln \eta$ for the polymerization of MMA with AIBN at $60^{\circ} \mathrm{C}$ in the mixed solvents of PEG400 and toluene at various ratios: $[\mathrm{MMA}]=1.0 \mathrm{~mol} \mathrm{I}^{-1} ;[$ AIBN $]=1.0 \times 10^{-2} \mathrm{moll}^{-1}$.

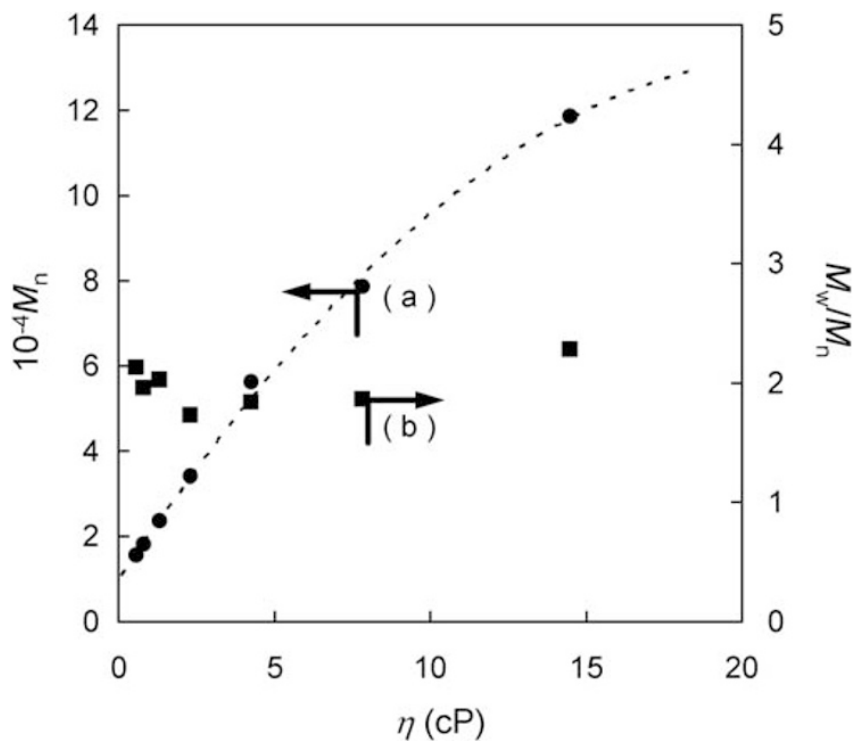

Figure 7 The $M_{\mathrm{n}}$ and $M_{\mathrm{w}} / M_{\mathrm{n}}$ values of PMMA obtained by polymerization with AIBN at $60^{\circ} \mathrm{C}$ in the mixed solvents of PEG400 and toluene at various ratios: $[M M A]=1.0 \mathrm{moll}^{-1}$; $[\mathrm{AIBN}]=1.0 \times 10^{-2} \mathrm{~mol} \mathrm{I}^{-1}$; (a) $10^{-4} \mathrm{M}_{\mathrm{n}}$ versus $\eta$; (b) $M_{\mathrm{w}} / M_{\mathrm{n}}$ versus $\eta$.

the linear plots of $\ln M_{\mathrm{n}}$ versus $\ln \eta$ is 0.65 (Figure 8). Although this value is slightly deviated from the theoretical value of 0.50 , the $M_{\mathrm{n}}$ value increases almost linearly with the square root of $\eta$ (Supplementary Figure S8). This result is also in good agreement with Equation (3).

The $0.5^{\text {th }}$-order dependence of both $R_{\mathrm{p}}$ and $M_{\mathrm{n}}$ on $\eta$ can be derived from the fact that the bimolecular termination rate is inversely proportional to $\eta$. 


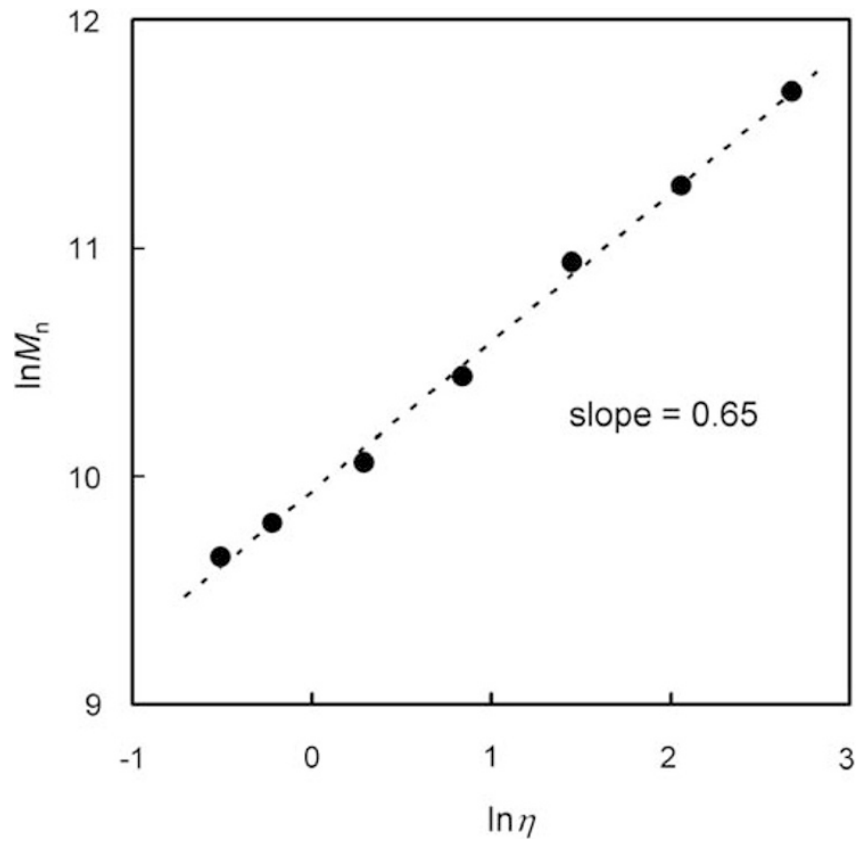

Figure 8 Plots of $\ln M_{n}$ versus $\ln \eta$ for the polymerization of MMA with AIBN at $60^{\circ} \mathrm{C}$ in the mixed solvents of PEG400 and toluene at various ratios: $[\mathrm{MMA}]=1.0 \mathrm{~mol} \mathrm{I}^{-1} ;[\mathrm{AIBN}]=1.0 \times 10^{-2} \mathrm{~mol} \mathrm{I}^{-1}$.

\section{Other aspects}

Because of the low coordination ability of PEG, it is highly unlikely that the ethylene glycol unit and the terminal alcohol of PEG can activate MMA like the Lewis acid, which is known to accelerate the FRP of MMA. ${ }^{43-45}$ The tacticity of PMMAs is not affected by the volume ratio of PEG400 to toluene in the mixed solvent and molecular weight of the PEG used; the triad syndiotacticity (rr) was constantly within $60.1-62.9 \%$. This also suggests that there is hardly such an interaction of PEG400 with the monomer and/or the polymer terminal.

Highly viscous media have been reported to reduce initiator efficiency by impeding the diffusion of primary radicals out of cage. ${ }^{24,37,46,47}$ However, it would be negligible in the PEG400 solvent because, as mentioned above, $R_{\mathrm{p}}$ has a linear relationship with the square root of the $\eta$ value. This clear relationship also suggests that the termination reaction would be hardly affected by the polymer chain length.

\section{Recovery and reuse}

Polymerization was carried out under the conditions of run 2 in Table 1. By the addition of ethanol into the polymerization mixture, PMMA was precipitated and filtered, and subsequently PEG400 was recovered by concentrating the filtrate. PEG400 was degassed in vacuo and reused for polymerization. This cycle was repeated three times, and PEG400 was finally recovered in 92\%. The PMMAs with $M_{\mathrm{n}}$ values of 130 000-140 000 were reproducibly obtained in $>90 \%$ yield. The PEG400 solvent can be recovered readily and reused without any loss of efficiency.

\section{Conclusion}

We have shown that the PEG solvent accelerates the FRP of MMA to quantitatively produce high-molecular-weight PMMA. This solvent system for FRP offers 'green', efficient and practical advantages. The kinetic results of the FRP in PEG400 demonstrate that both the $R_{\mathrm{p}}$ and $M_{\mathrm{n}}$ of the resulting PMMA are proportional to the square root of $\eta$. Therefore, it is reasonable that the highly viscous PEG400 solvent impedes translational diffusion of propagating radicals and consequently reduces the bimolecular termination rate. The diffusion of MMA, in contrast, is hardly suppressed. As a result, the diffusioncontrolled process increases the polymerization rate to produce highmolecular-weight PMMA. We suggest that the diffusion-controlled termination in PEG400 can be extended to other radical polymerizations; vinyl monomers with low polymerizability are now under examination. In addition, such a kinetic study as mentioned above is expected to be effective also for mechanistic elucidation of FRPs in newly emerging solvents such as ionic liquids and supercritical fluids.

\section{ACKNOWLEDGEMENTS}

This work was partially supported by the Rikougaku-Shinkoukai foundation (Tokyo Institute of Technology TLO). We thank Professor Bunichiro Yamada for helpful discussions.

1 Namboodiri, V. V. \& Varma, R. S. Microwave-accelerated Suzuki cross-coupling reaction in polyethylene glycol (PEG). Green Chem. 3, 146-148 (2001).

2 Li, J. H., Liu, W. J. \& Xie, Y. X. Recyclable and reusable Pd(OAc) $2 /$ DABCO/PEG-400 system for SuzukiMiyaura cross-coupling reaction. J. Org. Chem. 70, 5409-5422 (2005).

3 Liu, L., Zhang, Y. \& Wang, Y. Phosphine-free palladium acetate catalyzed Suzuki reaction in water. J. Org. Chem. 70, 6122-6125 (2005)

4 Yin, L., Zhang, Z. H. \& Wang, Y. M. PEG (300)-PdCl 2 promoted efficient and convenient Suzuki-Miyaura coupling of aryl chlorides with arylboronic acids. Tetrahedron 62, 9359-9364 (2006).

5 Li, J. H., Hu, X. C., Liang, Y. \& Xie, Y. X. PEG-400 promoted Pd(OAc) $2 / D A B C O-$ catalyzed cross-coupling reactions in aqueous media. Tetrahedron 62, 31-38 (2006).

6 Han, W., Liu, C. \& Jin, Z. L. In situ generation of palladium nanoparticles: a simple and highly active protocol for oxygen-promoted ligand-free Suzuki coupling reaction of aryl chlorides. Org. Lett. 9, 4005-4007 (2007).

7 Kumar, R., Chaudhary, P., Nimesh, S. \& Chandra, R. Polyethylene glycol as a non-ionic liquid solvent for Michael addition reaction of amines to conjugated alkenes. Green Chem. 8, 356-358 (2006).

8 Chandrasekhar, S., Prakash, S. J. \& Rao, C. L. Poly(ethylene glycol) (400) as superior solvent medium against ionic liquids for catalytic hydrogenations with $\mathrm{PtO}_{2}$. J. Org. Chem. 71, 2196-2199 (2006).

9 Zhou, H. F. Fan, Q. H., Tang, W. J, Xu, L. J., He, Y M., Deng G. J., Zhao, L. W., Gu, L. Q. \& Chan, A. S. C. Polyethylene glycol as an environmentally friendly and recyclable reaction medium for enantioselective hydrogenation. Adv. Synth. Catal. 348, 2172-2182 (2006).

10 Zhou, H. F., Fan, Q. H., Huang, Y. Y., Wu, L., He, Y. M., Tang, W. J., Gu, L. Q. \& Chan, A. S. C. Mixture of poly(ethylene glycol) and water as environmentally friendly media for efficient enantioselective transfer hydrogenation and catalyst recycling. J. Mol. Catal. A-Chem. 275, 47-53 (2007).

$11 \mathrm{Ma}$, X., Jiang, T., Han, B., Zhang, J., Miao, S., Ding, K., An, G., Xie, Y., Zhou, Y. \& Zhu, A. Palladium nanoparticles in polyethylene glycols: efficient and recyclable catalyst system for hydrogenation of olefins. Catal. Commun. 9, 70-74 (2008).

12 Santaniello, E., Manzocchi, A. \& Sozzani, P. Polyethylene glycols as host solvents: applications to organic synthesis. Tetrahedron Lett. 20, 4581-4582 (1979).

13 Chandrasekhar, S., Narsihmulu, C., Sultana, S. S. \& Reddy, N. R. Poly(ethylene glycol) (PEG) as a reusable solvent medium for organic synthesis. Application in the Heck reaction. Org. Lett. 4, 4399-4401 (2002).

14 Zhang, Z.- H., Yin, L., Wang, Y.- M., Liu, J.- Y. \& Li, Y. Indium tribromide in poly(ethylene glycol)(PEG): a novel and efficient recycle system for chemoselective deprotection of 1,1-diacetates. Green Chem. 6, 563-565 (2004).

15 Chandrasekhar, S., Narsihmulu, C., Saritha, B. \& Sultana, S. S. Poly(ethyleneglycol) (PEG): a rapid and recyclable reaction medium for the DABCO-catalyzed Baylis-Hillman reaction. Tetrahedron Lett. 45, 5865-5867 (2004).

16 Chen, J., Spear, S. K., Huddleston, J. G. \& Rogers, D. R. Polyethylene glycol and solutions of polyethylene glycol as green reaction media. Green Chem. 7, 64-82 (2005).

$17 \mathrm{Li}$, J. H., Zhu, Q. M., Liang, Y. \& Yang, D. Efficient and reusable $\mathrm{PdCl}_{2}(\mathrm{MeCN})_{2} / \mathrm{CuCl}_{2} /$ PEG-400 system for cyclization of alkenyl $\beta$-keto esters and amides. J. Org. Chem. 70, 5347-5349 (2005).

18 Heldebrant, D. J., Witt, H. N., Walsh, S. M., Ellis, T., Rauscher, J. \& Jessop, P. G. Liquid polymers as solvents for catalytic reductions. Green Chem. 8, 807-815 (2006).

19 Corma, A., Carcía, H. \& Leyva, A. Polyethylene glycol as scaffold and solvent for reusable C-C coupling homogeneous Pd catalysts. J. Catal. 240, 87-99 (2006).

20 Perrier, S., Gemici, H. \& Li, S. Poly(ethylene glycol) as solvent for transition metal mediated living radical polymerisation. Chem. Commun. 604-605 (2004). 
21 Yokota, K. \& Itoh, M. An improved kinetics for radical polymerization on the basis that the viscosity of the solvent plays an important role. J. Polym. Sci., Part B, Polym. Lett. 6, 825-830 (1968)

22 Yokota, K., Tomioka, H. \& Tazumi, A. Determination of chain transfer constant for solvent when the viscosity of the polymerizing system is considered important. J. Macromol. Sci.-Chem. A6, 1337-1347 (1972).

23 Matsumoto, A., Tanaka, S. \& Otsu, T. Synthesis and characterization of poly (1-adamantyl methacrylate): effects of the adamantyl group on radical polymerization kinetics and thermal properties of the polymer. Macromolecules 24, 4017-4024 (1991).

24 Matyjaszewski, K. \& Davis, T. P. Handbook of Radical Polymerization p 129-131, p 225, and p143 (John Wiley \& Sons, New York, 2002).

25 Trommsdorff, E., Köhle, H. \& Lagally, P. Zur polymerisation des methacrylsäuremethylesters. Makromol. Chem. 1, 169-198 (1947).

26 Benson, S. W. \& North, A. M. A simple dilatometric method of determining the rate constants of chain reactions. II. The effect of viscosity on the rate constants of polymerization reactions. J. Am. Chem. Soc. 81, 1339-1345 (1959).

27 North, A. M. \& Reed, G. A. Diffusion-controlled termination during the initial stages of free radical polymerization of methyl methacrylate. Trans. Faraday Soc. 57, 859-870 (1961).

28 North, A. M. \& Reed, G. A. Diffusion-controlled polymerization of some alkyl methacrylates. J. Polym. Sci., Part A 1, 1311-1321 (1963).

29 North, A. M. Diffusion-controlled reactions. Quart. Rev. 20, 421-440 (1966).

30 Bogunjoko, J. S. T. \& Brooks, B. W. Kinetics of free-radical polymerisation at high viscosities. Makromol. Chem. 184, 1603-1621 (1983).

31 Hong, K., Zhang, H., Mays, J. W., Visser, A. E., Brazel, C. S., Holbrey, J. D., Reichert, W. M. \& Rogers, R. D. Conventional free radical polymerization in room temperature ionic liquids: a green approach to commodity polymers with practical advantages. Chem. Commun. 1368-1369 (2002).

32 Harrisson, S., Mackenzie, S. R. \& Haddleton, D. M. Unprecedented solvent-induced acceleration of free-radical propagation of methyl methacrylate in ionic liquids. Chem. Commun. 2850-2851 (2002).

33 Harrisson, S., Mackenzie, S. R. \& Haddleton, D. M. Pulsed laser polymerization in an ionic liquid: strong solvent effects on propagation and termination of methyl methacrylate. Macromolecules 36, 5072-5075 (2003).
34 Strehmel, V., Laschewsky, A., Wetzel, H. \& Görnitz, E. Free radical polymerization of n-butyl methacrylate in ionic liquids. Macromolecules 39, 923-930 (2006).

35 Thurecht, K. J., Gooden, P. N., Goel, S., Tuck, C., Licence, P. \& Irvine, D. J. Free-radical polymerization in ionic liquids: the case for a protected radical. Macromolecules 41, 2814-2820 (2008).

36 Carswell, T. G., Hill, D. J. T., Londero, D. I., O'Donnell, J. H., Pomery, P. J. \& Winzor, C. L. Kinetic parameters for polymerization of methyl methacrylate at $60^{\circ} \mathrm{C}$. Polymer 33 , 137-140 (1992).

37 Shen, J., Tian, Y., Wang, G. \& Yang, M. Modelling and kinetic study on radical polymerization of methyl methacrylate in bulk, 1 . Propagation and termination rate coefficients and initiation efficiency. Makromol. Chem. 192, 2669-2685 (1991).

38 Carswell, T. G., Hill, D. J. T., Hunter, D. S., Pomery, P. J., O'Donnell, J. H. \& Winzor, C. L. Simultaneous measurement by ESR spectroscopy of monomer conversion and radical concentration during polymerization for determination of kinetic parameters. Eur. Polym. J. 26, 541-544 (1990).

39 Shen, J. C., Wang, G. B., Yang, M. L. \& Zheng, Y. G. A kinetic study on MMA bulk radical polymerization. Polym. Int. 28, 75-79 (1992).

40 Balke, S. T. \& Hamielec, A. E. Bulk polymerization of methyl methacrylate. Appl. Polym. Sci. 17, 905-949 (1973).

41 Burnett, G. M. \& Duncan, G. L. High conversion polymerisation of vinyl systems. I. Methyl methacrylate. Makromol. Chem. 51, 154-170 (1962).

42 Jiang, S., Sudol, E. D., Dimonie, V. L. \& El-Aasser, M. S. Kinetics of dispersion polymerization of methyl methacrylate and $n$-butyl acrylate: effect of initiator concentration. Macromolecules 40, 4910-4916 (2007).

43 Imoto, M., Otsu, T. \& Harada, Y. Vinyl polymerization. LXVIII. The polymerization of methyl methacrylate in the presence of zinc chloride. Makromol. Chem. 65, 180-193 (1963).

44 Yamada, B., Kusuki, Y. \& Otsu, T. Radical polymerizations of vinyl monomer-stannic chloride complexes. Makromol. Chem. 137, 29-42 (1970).

45 Bamford, C. H., Brumby, S. \& Wayne, R. P. Effect of zinc chloride on the velocity coefficients in the polymerization of methyl methacrylate. Nature 209, 292-294 (1966).

46 Schrijver, F. D. \& Smets, G. Polymerization kinetics in highly viscous media. J. Polym. Sci., Part A-1 4, 2201-2210 (1966).

47 Russell, G. T., Napper, D. H. \& Gilbert, R. G. Initiator efficiencies in high-conversion bulk polymerizations. Macromolecules 21, 2141-2148 (1988).

Supplementary Information accompanies the paper on Polymer Journal website (http://www.nature.com/pj) 